\title{
The optimized shape of a leaf petiole
}

\author{
D. Pasini \& V. Mirjalili \\ Department of Mechanical Engineering, McGill University, \\ Montreal, Canada
}

\begin{abstract}
A plant leaf is generally composed of a petiole and a leaf blade. The petiole connects the leaf blade to the plant stem and, from a structural viewpoint, it resembles a cantilever beam. Petiole design is driven by the minimum use of material to withstand a combined torsion and bending load. The cross-section has a transverse size decreasing lengthwise and has a grooved shape. This paper examines the structural efficiency of the petiole shape. Ten petiole specimens of dicotyledonous plants have been investigated. Continuum mechanics and dimensionless factors are used to model the stiffness properties of the petioles. The results of the characterization are visualized on maps that contrast petiole efficiency to that of reference cross-sections. Nature shapes the petiole material to secure the best trade off between torsional compliance and flexural stiffness.

Keywords: leaf petiole, structural efficiency, optimized shape, torsional compliance, bending stiffness.
\end{abstract}

\section{Introduction}

Plants are complex systems that perform several vital functions. Their organs work in synergy to govern a variety of tasks, such as mineral absorption, water supply, photosynthesis, food storage, and structural support. During growth, the plant organs that interface with the environment receive different stimuli from light, gravity, touch, as well as from change of soil salinity and stress concentration. As a response to these factors, the plant adapts its morphology.

Plants have a large variety of colours, forms and size, which make them attractive. Appearance, though, is just one among the remarkable features. Plants are designed to multi-function as well as to support a variety of loading configurations by using the least amount of bio-material [1]. 
This paper focuses on one specific organ of plants, the leaf petiole. From a vital point of view, the leaf is one of the crucial parts of a plant. Through photosynthesis, a plant processes the sun's energy to produce its own sugar [2]. However, from an engineering point of view, the leaf has a noteworthy structure, in particular its petiole. The shape of this organ is remarkable, for it permits an efficient support of loading. For example, it allows the leaf to swing freely in the wind in order to reduce the aerodynamic forces; it supports the weight of the leaf blade, as well as any moisture, rain, snow, and insects; it also enables the leaf blade to twist towards the sunlight and catch the sun's rays. To function with the least amount of material, the petiole exploits the material anisotropy and tailors the shape properties of its body [3-7].

This paper examines the structural efficiency of the petiole to bear combined loading. The aim is to look into the performance of its cross-sectional shape. The first part of the paper describes organs and functions of plants in order to put the petiole into the biological context. Then, the factors that stimulate shape changes are reviewed. In the last sections, the analysis is narrowed to the stalk structural design. Ten petiole specimens of dicotyledonous plants have been examined, and their efficiency compared to that of reference shapes. The analysis hinges on dimensionless parameters that are used for modeling and developing performance charts. The maps help to gain insight into the biological design of the leaf petiole.

\section{Plant body and organs}

Angiosperms are flowering plants where reproductive organs are within flowers, and seeds are in the fruits. The number of seeds, or cotyledons, is used to sort angiosperms into two classes: the monocots, which have a single cotyledon, and the dicots with two seeds. Despite the differentiation, three elements are common to all angiosperms: roots, stems, and leaves. These are grouped into two systems: the root and the shoot, which consists of stem and leaves (Fig. 1).

Root System. The main vital functions of roots are the absorption of water and minerals, their conduction from the roots to the stem and vice versa, and lastly, the storage of food. The structural function of the roots is to provide anchor in the soil (Fig.1). An analogy in engineering is bolts that hook a column firmly in a foundation. Whereas monocots develop a fibrous roots system, in dicots a major root grows vertically and it is called taproot. This structural diversity is reported in Table 1 together with other differences between the classes.

Shoot System. The stem supporting the leaves is the shoot system. Attached at nodes through petioles, leaves form an angle with the stem (Fig.1). Here, there are also axillary buds that can develop, although most of them are dormant. At the terminal top, an apical bud is partly responsible for inhibiting the growth of axillary buds. The phenomenon, called apical dominance, occurs because the plant has to increase exposure to light, especially in a location with dense vegetation [2]. The other constituents of the shoot are the photosynthetic organs. Although their form, size, and even colour, can vary, a leave is generally 
structured as a flatten blade, connected to the stem by a petiole, or stalk. Some monocots, however, lack petioles, such as in grasses.

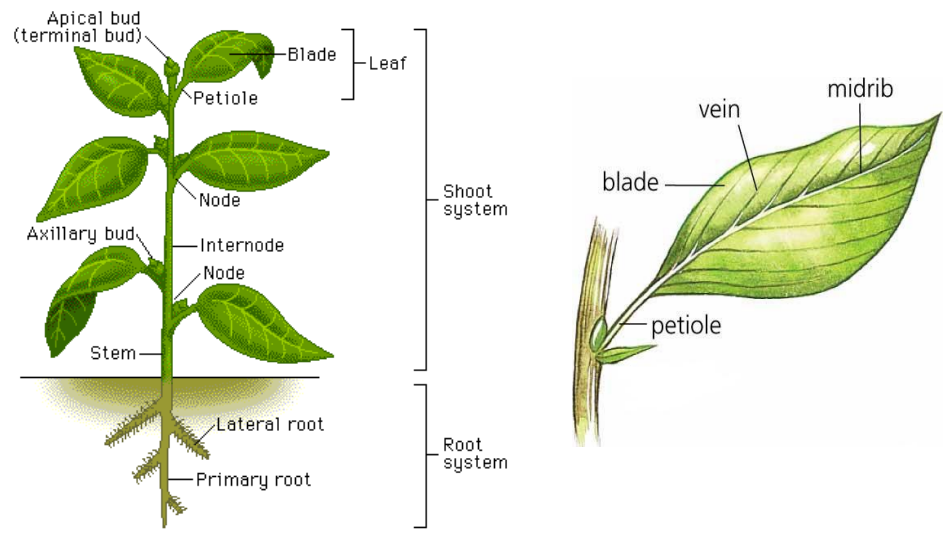

Figure 1: The structure of an angiosperm (right) and a zoom of its leaf (left) [2].

Table 1: $\quad$ A comparison of monocots and dicots [2].

\begin{tabular}{|c|c|c|}
\hline & Monocots & Dicots \\
\hline Embryos & One cotyledon & Two cotyledons \\
\hline Leaf Venation & $\begin{array}{l}\text { Veins usually parallel } \\
\text { (e.g. palms) }\end{array}$ & $\begin{array}{l}\text { Veins usually netlike } \\
\text { (e.g. maples) }\end{array}$ \\
\hline Roots & Fibrous root system & Taproot system \\
\hline Stems & $\begin{array}{l}\text { Vascular bundles usually } \\
\text { complexly arranged }\end{array}$ & $\begin{array}{l}\text { Vascular bundles usually } \\
\text { arranged in a ring }\end{array}$ \\
\hline Flowers & $\begin{array}{l}\text { Floral parts usually in } \\
\text { multiples of three }\end{array}$ & $\begin{array}{l}\text { Floral parts usually in } \\
\text { multiples of four or five }\end{array}$ \\
\hline Growth & $\begin{array}{l}\text { No secondary growth / annual } \\
\text { ring formation }\end{array}$ & $\begin{array}{l}\text { Secondary growth / annual } \\
\text { ring formation }\end{array}$ \\
\hline
\end{tabular}

\section{Plant tissue systems}

The tissue is a cluster of similar cells into units. Three tissue systems grow continuously throughout a plant: dermal, vascular, and ground tissue. New tissues are formed at the meristems, the growing points on a plant.

Dermal System. The dermal tissue system is the epidermis. Analogous to our skin, the dermal tissue is a layer of tightly packed cells that protect plant organs. It is covered by a waxy coating, called cuticle, which reduces water loss through evaporation. This protection is imperative during dry summers. The pores, called stomata, control the exchange of gases between plant and surroundings. If a plant 
undergoes secondary growth, the epidermis is replaced by dead waterproofed cells, the peridermis.

Vascular System. Xylem and phloem are two vascular systems. The former conveys water and minerals from roots into the shoots. The latter provides structural support and is in charge of transporting food from mature leaves to the roots, as well as to parts of the shoot system, e.g. developing leaves and fruits.

Ground System. Beyond photosynthesis, storage, and support, the ground tissue governs the metabolic processes. It is mainly made up of thin walled cells forming the parenchyma. This tissue fills the space between the dermal and vascular ones and makes up the plant bulk.

\section{Plant movements}

Plant movements change the body shape of an organ. Two types are the tropisms and the turgor. The former is a response of the plant curvature to stimuli. If toward the stimuli, the tropism is positive, whereas it is negative when the move is away from it. On the other hand, the turgor movements are rapid and reversible, often triggered by pressure changes in the state of the cells subject to stimuli.

\subsection{Tropisms}

Phototropism is the bending of a plant either towards or away from a light source. It ensures that photosynthesis will take place.

Gravitropism is the response of a plant to gravity. When it is positive, the roots grow deep into the soil to secure water and other nutrients. If negative, the shoots develop upwards to the sunlight for the photosynthesis.

Thigmotropism is a directional growth in reaction to touch. Unlike stems that grow straight, vines, for example, have tendrils that coil at touch with an object.

\subsection{Turgor movements}

Rapid leaf movements. Under strong winds, the leaves of the mimosa and other plants collapse and fold one upon another. It is speculated the rapid move helps plants retain water by reducing the surface area of the leaf.

Sleep movements are responses to changes of light during the course of the day. Legumes and bean plants, for example, raise their leaves horizontally in the morning, and then lower them vertically at the sunset.

\section{Plant responses to environmental stresses}

Besides morphological adaptation, plants modify their shape also in response to environment changes. For example, a water deficit stimulates the synthesis of a hormone, i.e. abscisic acid, which induces the pore closure and reduces evaporation. As a result, cells loose turgor and expose less surface to sun. 
Oxygen deprivation is another cause that accelerates the growth of air tube cells in certain roots to ease oxygen supply.

Other triggers for shape changes are cold, heat, salt, and compressive stresses. Cold temperature makes the plant to increase its proportion of unsaturated fatty acids. Heat stress, above a certain temperature, stimulates the production of special proteins, called heat-shock proteins. Excess of sodium, also, threatens plant grow. Salt can cause root to loose water even though the soil is submerged in water. This occurs when the osmotic pressure of the surrounding water is more negative than that of the root tissue.

Lastly, the other important shape-adaptation is triggered by compressive stresses. In response to these, a plant modifies its size and shape. Cells grow in regions exposed to compressive stresses that make the thin-walls of the material micro-structure unstable. This sensitivity of shape to buckling stress is the reason why, for a plant, strength in compression is lower than strength in tension.

\section{The leaf petiole}

Previous sections have examined the vital functions of a plant, its organs and the triggers for morphological changes. This section zooms into one of its organ, the leaf petiole, and it examines its shape efficiency. This is not intended to suggest that only shape efficiency matters; all the other vital functions are essential, but they are not the focus here. The analysis is based on classic mechanics, although its limits when applied to the biological world. Before efficiency modelling, functions, morphology and bio-material of the petiole are described.

Loadings. The structural functions of a leaf resemble those of a cantilever beam. The petiole provides appropriate bending stiffness to support surface loads, such as the blade weight, as well as any other, whether it be rain, snow, moisture, or even the weight of an insect. But bending is not the only loading. The aerodynamic force of the wind causes the petiole to twist. In addition, the petiole coils to reach and to respond to sunlight. Hence, a combination of bending and torsion can be assumed as the overall loading.

Structural morphology. Fig. 1 illustrates the petiole connecting the leaf blade to the stem. In response to compressive stresses induced by the coupled load, the petiole results in being flexible, as opposed to conventional engineering cantilevers, which are designed to be stiff. The petiole is tapered lengthwise and it has often an asymmetric cross-section, grooved at its top. Such a shape lowers torsional stiffness without compromising the deflection resistance to gravity loading. The benefit of the groove is twofold. First, an increase of twist flexibility allows the leaves to bunch and reduce wind drag. Second, the leaf can orient itself towards downwind and even reduce the requirement for flexural stiffness.

Bio-material. Increasing torsion compliance without compromising resistance in bending is achieved by exploiting not only shape properties, but also material. Unlike most of engineering materials, petiole microstructure is inhomogeneous and anisotropic. It is made up of thick liquid cells supporting compression at the petiole bottom, and it has thin elongated cells at its top to 
bear tension. Current biological models, however, find difficult to explain how anisotropy lowers torsion stiffness relative to bending stiffness [1].

The next section aims at obtaining insight into the macro-shape of the petiole. We are interested in how shape and size are tailored to better the petiole response to the combined load.

\section{Case study: structural performance of dicotyledonous petioles}

The petiole cantilever undergoes mainly the coupling of torsion and bending. Its structural response exploits flexibility, rather than stiffness. This section examines the cross-section efficiency at the stem node, where max load occurs. As performance index, we consider the ratio flexural to torsional stiffness.

10 specimens of dicots petioles (Table 2) have been analysed, and their shape performance has been examined. To compare their efficiency with those of elliptical and semi-elliptical cross-sections, we resort on dimensionless measures, called Shape Transformers [8]. A shape transformer, $\psi_{g c}$, is defined for a geometric quantity, $g c$, of a cross-section. It is obtained by normalizing $g c$ to $g c_{D}$, which is the quantity of the rectangular Envelope described by the crosssection size. Shape transformers can govern the geometry metrics of a shape regardless of its size. For example, shape transformers for the area and second moment of area are $\psi_{A}=A / A_{D}$ and $\psi_{I}=I / I_{D}$. The normalization of the geometry enables to decouple the contribution of the shape to that of the Envelope.

The following examines the structural performance that the petiole crosssection exhibits to improve torsion flexibility without loosing bending resistance. A way to describe this criterion of excellence is to consider the flexural to torsion stiffness ratio, such that efficiency is optimised by maximising the index:

$$
p=\frac{E I}{G J}=\frac{E I_{D} \psi_{I}}{G J_{D} \psi_{J}}
$$

where $E$ and $G$ are Young's and shear Modula, $\psi_{I}$ and $\psi_{J}$ are the transformers of second moment of area and the polar moment, and $I_{D}$ and $J_{D}$ are the quantities of the envelope. Table 2 reports $\psi_{I}$ and $\psi_{J}$ for the petiole specimen, and Table 3 lists those for solid and hollow ellipses and semi-ellipses.

Three design scenarios are now examined. First, we characterize the flexural stiffness of the cantilever; second, its torsional rigidity; and lastly, its efficiency described by the index (1). The results are displayed on efficiency maps that help gain insight into the biological design of the petiole.

\subsection{Flexural stiffness}

To characterize the flexural stiffness, $\psi_{I}$ expressions from Table 3 have been plotted against $\psi_{A}$ in Fig. 2. The coordinates of a point represent the bending stiffness of a cross-section shape for a given volume and regardless of material. Shapes that are stiff lie on the top left as opposed to those that have low $\psi_{I} / \psi_{A}$ and lie on the bottom right. The chart illustrates two distinct domains of 
properties. One encloses the ellipses classes and it is bordered by curves $1 \mathrm{e}$ and $2 \mathrm{e}$. The other is for semi-ellipses and is bounded by curves $1 \mathrm{~s}$ and $2 \mathrm{~s}$. Regardless of the envelope, each curve describes the flexural stiffness with respect to fraction and location of material within the envelope. For a given $\psi_{A}$, the ellipses' classes are not as stiff as the semi-ellipses, for the asymmetry permits the material to be placed far from the neutral axis. Specimen shape transformers from Table 3 have been plotted in Fig.2. The plot shows that petioles' shapes are quite efficient in providing flexural stiffness, considering their solid shape. Petioles n. 3,7,10 exhibit the highest resistance to bending deflection.

Table 2: $\quad$ Geometry of dicots petiole specimen.

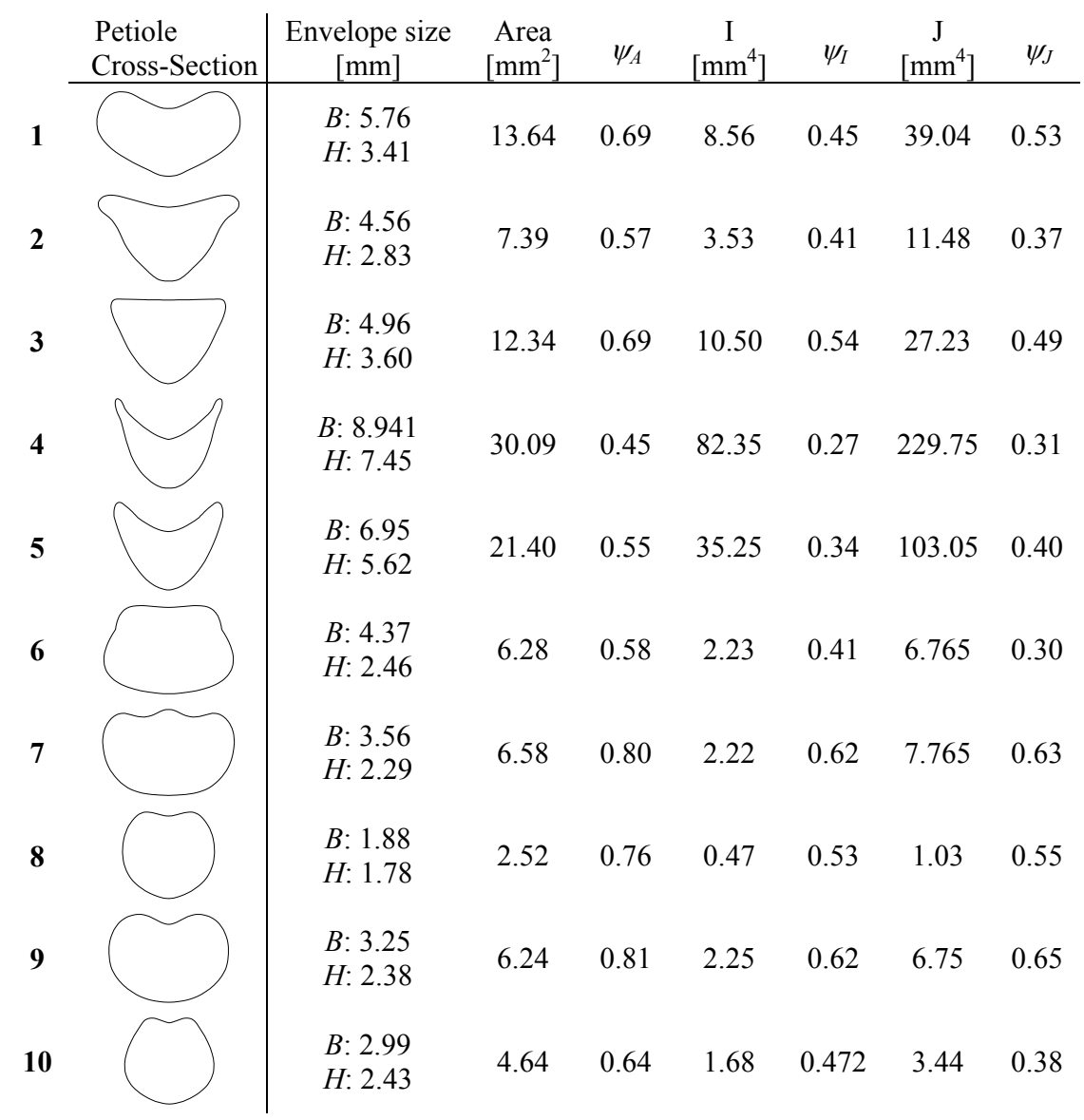


42 Design and Nature III: Comparing Design in Nature with Science and Engineering

Table 3: $\quad$ Shape transformers and efficiency parameters.

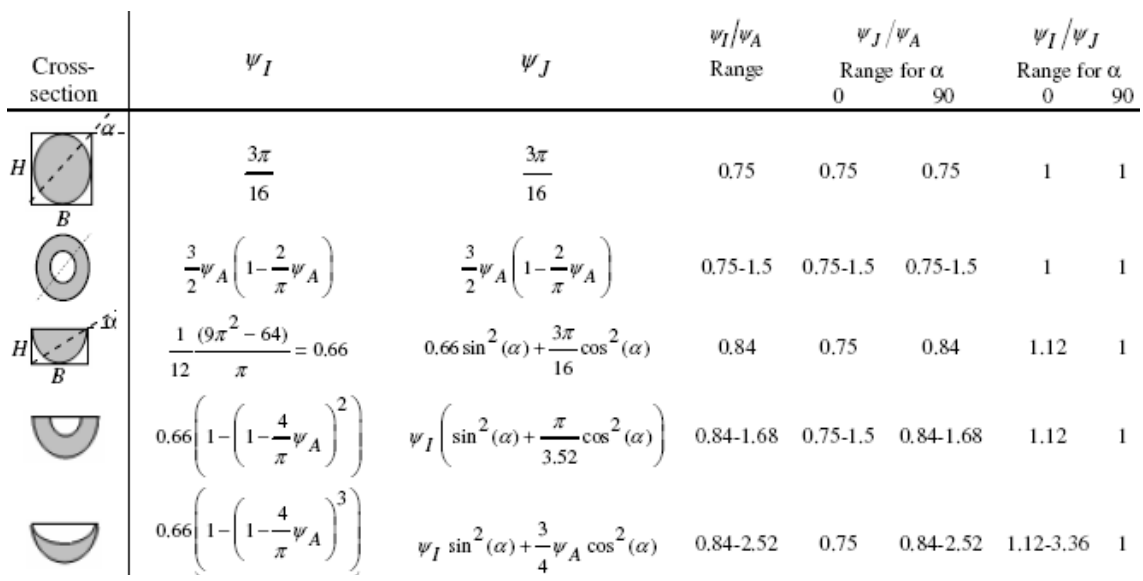

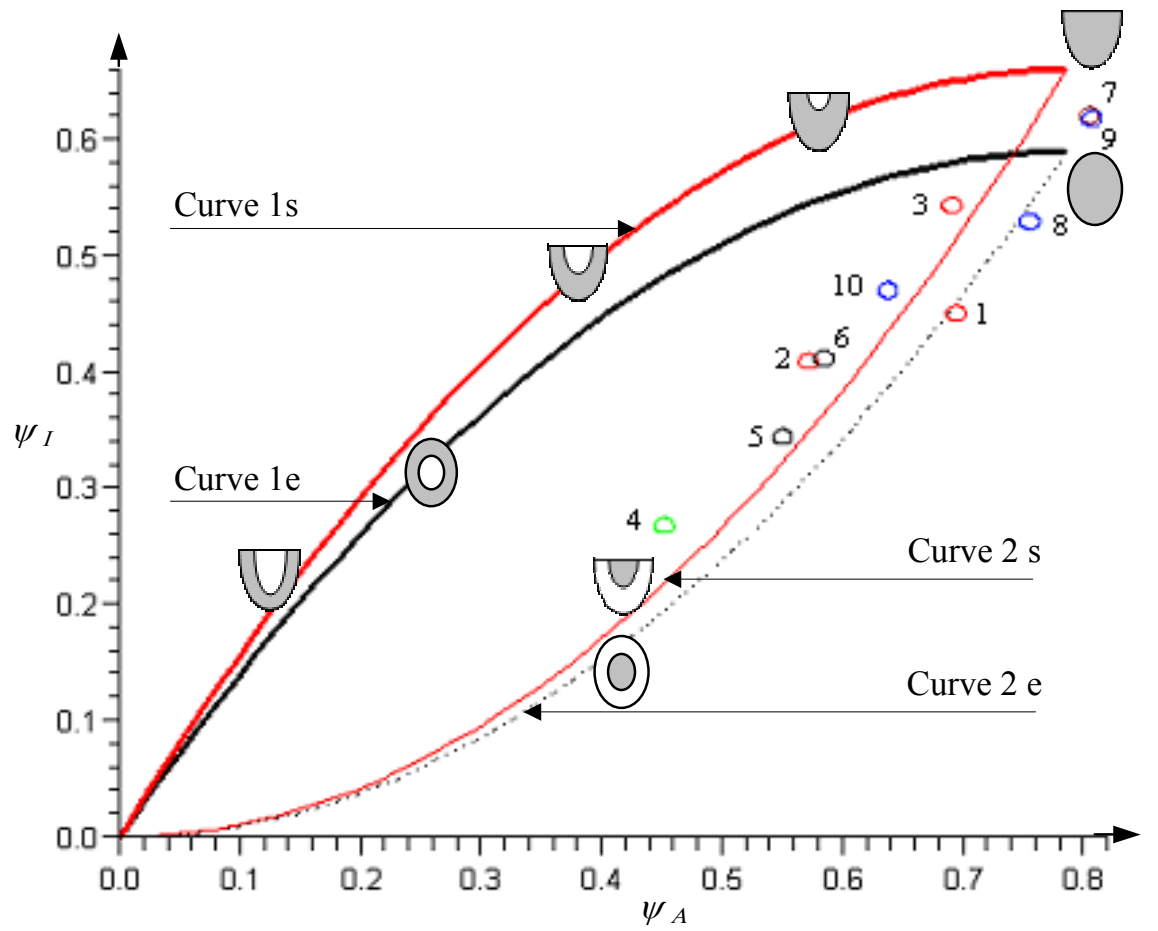

Figure 2: $\quad$ Flexural stiffness for specimen, ellipses and semi-ellipses classes. 


\subsection{Torsional stiffness}

Unlike flexural shape transformers, $\psi_{J}$ for asymmetric shapes depends on the ratio of the envelope size. Table 3 shows that for envelope stretched width-wise with $\alpha \rightarrow 0$, the semi-ellipses class is as stiff as that of the ellipses one. This is also illustrated in Fig. 3, where $\psi_{J}$ is plotted versus $\psi_{A}$. The properties domain of the ellipses class is described by the same curves of the semi-ellipses class., i.e. curve $1 \mathrm{e} \equiv$ curve $1 \mathrm{~s}(\alpha \rightarrow 0)$ and curve $2 \mathrm{e} \equiv$ curve $2 \mathrm{~s}(\alpha \rightarrow 0)$. However, for $\alpha>0$, there is a shift of the semi-ellipses domain upwards to the limit $\alpha \rightarrow 90$. This means that the ellipses' classes shown in Figure 3 are easier to twist. On the other hand, the semi-ellipses are stiffer for increasingly deeper envelopes.

We now contrast the domain properties with the Shape Transformers of the specimens. The petioles show low torsional stiffness although their shapes are asymmetric. Petioles n. 2,6,10 are even more compliant than the ellipses and semi-ellipses of the lower bound. As expected, petiole design is flexible; the leave can sway back and forth in order to reduce drag force and to reach sunlight.

\subsection{Petiole scenario: torsion and bending coupling}

Figure 4 illustrates the relation of $\psi_{I}$ to $\psi_{J}$ (Tables 2 and 3). The index 1, used for efficiency comparison, is the slope of the line a point forms with the origin. For a given flexural stiffness, the higher the slope, the easier it is to twist the shape.

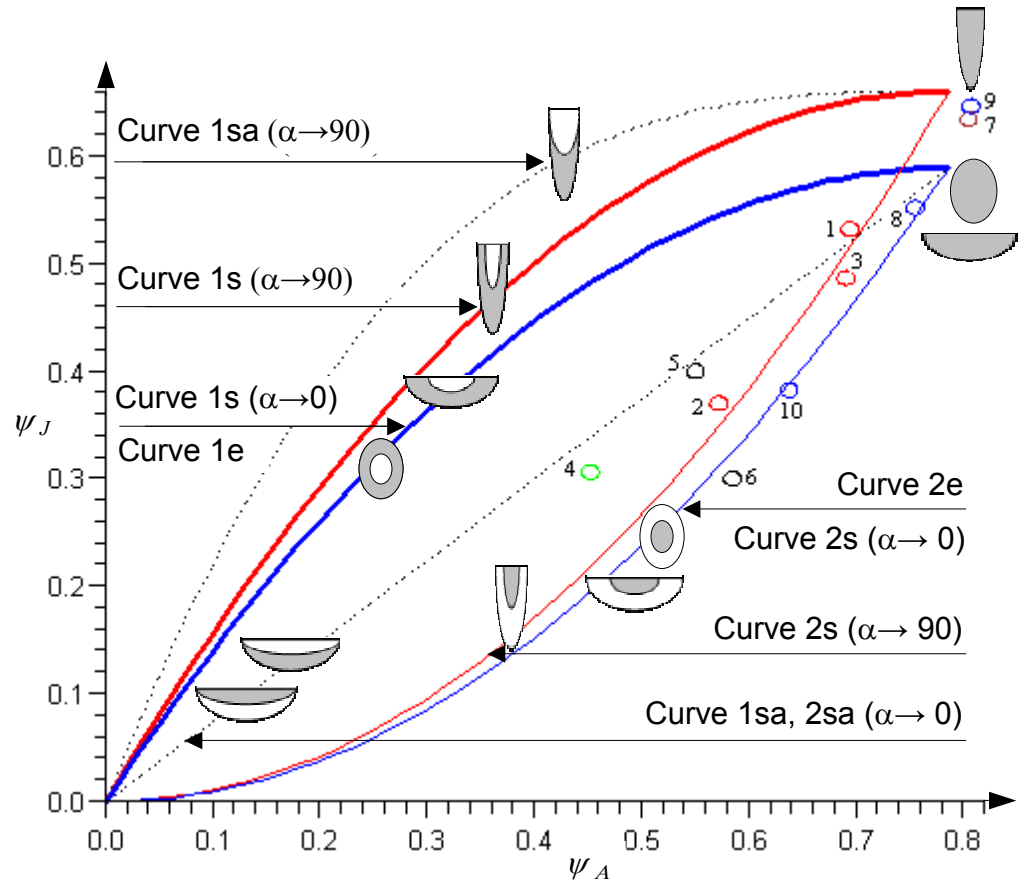

Figure 3: Torsional stiffness for specimen, ellipses and semi-ellipses classes. 


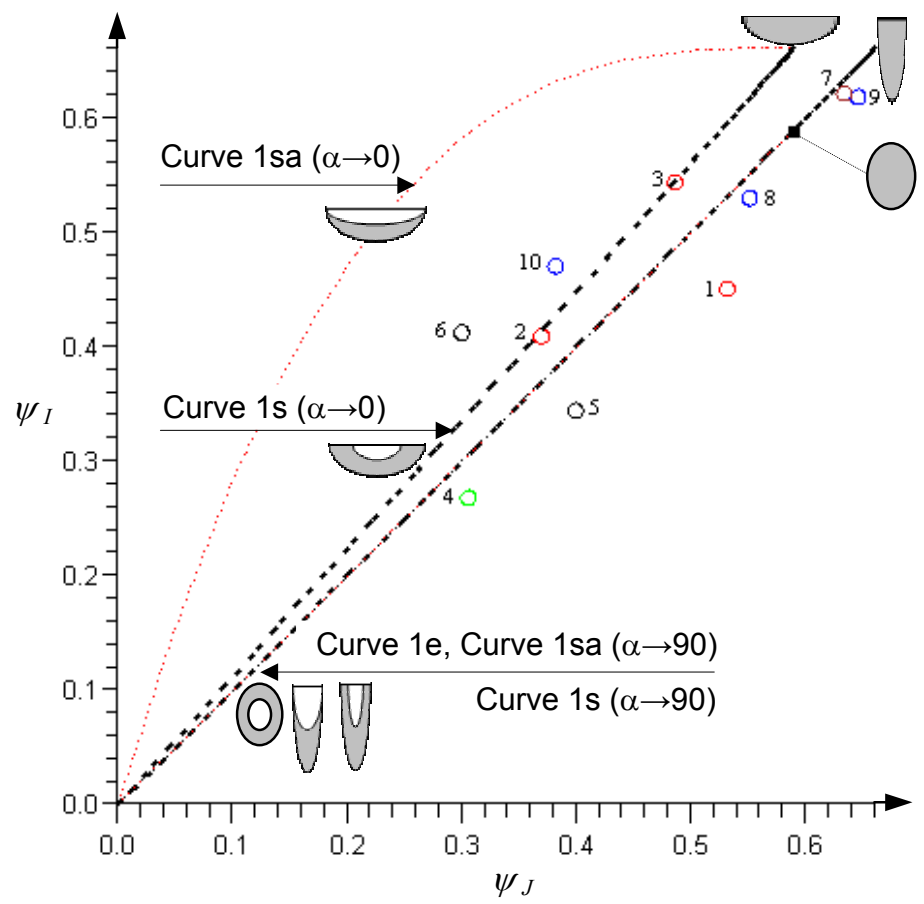

Figure 4: Flexural vs. Torsional stiffness for specimen, ellipses and semiellipses classes.

The map bisector describes shapes with same torsional and bending stiffness. These concepts are hollow ellipses as well as very deep semi-ellipses $(\alpha \rightarrow 90)$. They are the best trade-off for both bending and torsion stiffness. However, stretching the semi-ellipse widthwise lowers the torsional stiffness. The curve $1 \mathrm{~s}$ $(\alpha \rightarrow 90)$ shifts left to curve $1 \mathrm{~s}(\alpha \rightarrow 0)$ for proportionally scaled layers, whereas it moves further up to the curve 1 sa $(\alpha \rightarrow 0)$ for the other class of the semi-ellipses. Envelope changes, on the contrary, do not improve efficiency for symmetric shapes. The map highlights that lowering torsion stiffness by widening the envelope width has the impact of reducing the requirement for flexural stiffness.

The plot of specimens properties in Figure 4 illustrates petioles n. 2, 3, 6, 10 lie between curve $1 \mathrm{~s}(\alpha \rightarrow 0)$ and curve 1 sa $(\alpha \rightarrow 0)$. Though solid, the shapes are better than the proportionally scaled semi-ellipses. They are stretched widthwise, and their shapes best resemble the semi-ellipses class shown at base of table 3 .

Petiole design is optimized at different length scales. The maps presented in this section aim at gaining insight into the biological design of macro-shapes. However, the impact that material anisotropy has on petiole performance cannot be undervalued, even though current biological models strive to explain how Nature tailors $G$ and $E$ at the micro-scale. 


\section{Conclusions}

The leaf petiole has a large number of functions. Its structure is optimized to bear mainly a combination of bending, due to gravity load, and torsion, induced by wind drag. This paper has examined the structural efficiency of petiole shapes. A case study of 10 dicotyledonous petioles has been carried out to gain insight into shape performance. Efficiency maps have been developed to contrast biological design to conventional engineering cross-sections. The charts show that size and shape of the petiole are exploited to ease the twist as well as to lower the requirement of flexural stiffness.

\section{Acknowledgement}

The authors thank Mrs. Stephanie Rinaldi, undergraduate of McGill University, for the collection of specimen data.

\section{References}

[1] Vogel, Steven. Comparative Biomechanics: Life's Physical World. Princeton University Press, 2003.

[2] Campbell, Neil A., Jane B. Reece, and Lawrence G. Mitchell. Biology. Fifth Edition. Don Mills: Benjamin Cummings, 1999.

[3] Ennos, A.R., The mechanics of the flower stem of the sedge carex acutiformis. Annals of Botany, 72(2), pp. 123-127, 1993.

[4] Etnier, S. A. and Vogel, S., Reorientation of daffodil (Narcissus: Amaryllidaceae) followers in wind: drag reduction and torsional flexibility. American Journal of Botany, 87, pp. 29-32, 2000.

[5] Ennos, A.R., Spatz, H.C. and Speck, T., The functional morphology of the petioles of the banana, Musa textilis. J. of Experimental Botany, 51, pp. 2085-93, 2000.

[6] Etnier, S. A., Flexural and torsional stiffness in multi-jointed biological beams. Biological Bulletin, 200, pp. 1-8, 2001.

[7] Vogel, S., Twist-to-bend ratios of woody structures. J. of Experimental Botany, 46, 1995.

[8] Pasini, D., Shape Transformers for Material and Shape Selection, ASME DETC-84894, 2005. 